\title{
La Planificación turística, enfoque de la calidad en los servicios
}

\author{
Tourism Planning, focus on quality services \\ Christian Rivera García. ${ }^{1}$, Jesica Mariana Cachipuendo Castillo. ${ }^{2}$
}

\section{DOI: https://doi.org/10.33262/visionariodigital.v3i4.970}

\begin{abstract}
The integration of the elements of tourism planning, increases the chances of visiting a country, working with multisectoral needs that facilitates the identification of problems in the area of recreation and leisure, strategies are atomized to meet the needs of travelers in the quality of services, but increasing the processes of innovation and tourism promotion as endogenous and exogenous elements of planning, which responds to systematic thinking, in an organized way, allows future projections, explains possibilities in prefeasibility and feasibility, proposes objectives to Short, medium and long term, it analyzes advantages and disadvantages, which allow the detection of opportunities in consideration of short-term premises of participation in co-responsibility of alternatives according to previously established objectives and goals in response to the plans, programs and projects linking heterogeneous the budgets of co-participation, in this field the investigation assumes the objective of planning the tourist activity in an integral way to propose a progressive change in the quality of the services, that impel the national and foreign investment in increase of travelers interested in the nature and culture being held to security processes and protocols without contrasting production methods and consumption patterns, in the framework of reducing negative impacts on the Ecuadorian geographical tourist territory.
\end{abstract}

Words Keys: Planning, tourism planning, services, quality

\section{Resumen}

La integración de los elementos de la planificación turística, incrementa las posibilidades de visita a un país, se trabaja con necesidades multisectoriales que facilita la identificación de problemas en el área de recreación y ocio, las estrategias se atomizan para satisfacer las necesidades de los viajeros en la calidad de los servicios, pero incrementando los procesos de innovación y promoción turística como elementos endógenos y exógenos de la planificación, que responde al pensamiento sistemático, de manera organizada, permite realizar proyecciones a futuro, explica posibilidades en prefactibilidad y factibilidad,

1 Universidad Técnica de Babahoyo, Ecuador, crivera@utb.edu.ec

2 Universidad Técnica de Babahoyo, Ecuador, jcachipuendo@utb.edu.ec 
propone objetivos a corto, mediano y largo plazo, analiza ventajas y desventajas, que posibiliten la detección de oportunidades en consideración de premisas coyunturales de participación en corresponsabilidad de alternativas de acuerdo a objetivos y metas previamente establecidas en respuesta a los planes, programas y proyectos vinculando de manera heterogénea los presupuestos de coparticipación, en esta ámbito la investigación asume el objetivo de planificar integralmente la actividad turística para proponer un cambio progresivo en la calidad de los servicios, que impulse la inversión nacional y extranjera en incremento de viajeros interesados por la naturaleza y cultura sujetándose a procesos y protocolos de seguridad sin contrastar los métodos de producción y los patrones de consumo, en el marco de disminuir los impactos negativos sobre el territorio geográfico turístico ecuatoriano.

Palabras claves: Planificación, planificación turística, servicios, calidad

\section{Introducción}

El turismo siempre está relativizando sus actividades en el marco de su filosofía y el contexto mundial en necesidades de recreación y ocio del turista, este entramado sobrepasa el la responsabilidad autónomo, asumido como responsabilidad integral con plena participación social comunitaria, en este trabajo se consideró la "investigación" como una acto humano sistemático y organizado destinado a producir conocimientos y a la "planificación turística" como la guía de pensar antes de actuar, utiliza procesos para introducir una mayor racionalización y organización en las actividades y acciones propias de la actividad turística, que debe alcanzar sus objetivos y metas preestablecidas de manera eficiente y eficaz, maneja los recursos que en algunos casos son escasos y en otras puestos a prueba. El enfoque de desarrollo sostenible filtra parámetros participativos, rescatados de la actividad, especialmente urbana, interesada en descontextualizar la realidad en su entorno participativo permanente, ha sembrado la necesidad de aprovechamiento racional de los recursos naturales y culturales, no como dueños sino como elemento adjunto a la cosmovisión de la sabiduría humana. La sostenibilidad nos ha conducido al desaprovechamiento de estrategias muy bien diseñadas y planificadas, pero sin resultados óptimos e insuficientemente satisfactorios; particularmente desde el punto de vista de la integración corresponsable sumergidos en una ecuación: para los que piensa por otros y los que piensan por sí mismos, desconecta la interrelación turística y ambiental con la población presente y futura, adicionalmente se puede afirmar que, el desarrollo está inmerso en la planificación que ha permitido libertad de decisiones y aseguramiento cognitivo, actitudinal y procedimental en los servicios turísticos que ofrece un destino en particular.

La multi sectorialidad poco orientada a resolver los problemas prioritarios del país, ha encontrado en la planificación e implementación de programas o proyectos directamente vinculados con la producción y exportación, la imbricación activa de las universidades, empresa privada, instituciones de ciencias y tecnología, organizaciones privadas innovadoras y profesionales del turismo para alcanzar la verdadera libertad de elegir como queremos atraer la inversión extranjera, el incremento de turistas, mejorar los servicios, bienes y productos, 
frente al mercado nacional, regional e internacional en la satisfacción de necesidades de recreación.

Satisfacer las necesidades del hombre es un reto impostergable para una sociedad con pleno auge tecnológico, esto ha producido el mejoramiento, incalculable en la vida de ciertas sociedades planetarias que tecnifican sus vidas para optimizarlas. Los viajes tecnológicos ya no son una barrera, son una realidad, que verifica la ambición humana en la expansión y la conquista de nuevos campos. Actualmente se planifican viajes a la luna para observar nuestro planeta desde el espacio se considera esta actividad como una especie de turismo espacial. El turismo siempre ha estado involucrado con la actividad del hombre en todos los ámbitos, cambiando el desarrollo económico y cultural, aunque ensimismado y por destacar, aún en lo ecológico.

Las grandes urbes donde se práctica la actividad turística a gran escala como: la India, China, Estados Unidos, Alemania, Francia, entre otras, tuvieron que pasar por grandes transformaciones, consideraciones y adaptaciones a los nuevos perfiles de los turistas, estas son sus principales armas la planificación futurista y los proyectos ambiciosos de uso racional de la cultural y procesos ecológicos internos, todo esto bajo la premisa de "conservación". Organizar las actividades turísticas para el disfrute y distracción de viajeros nacionales y extranjeros, es plenamente reconocida como planificación, estar un paso adelante de las necesidades del viajero es clave de éxito, planear los bienes y servicios de uso y consumo es una ventaja de la planificación turística, que se refleja en el aumento considerable del número de viajeros que visitan lugares posicionados estratégicamente en el mercado de viajes y destinos.

Se considera las nuevas tendencias turísticas mundiales, América Latina es el mercado del descanso natural y de la salud integral, que presentan las comodidades particularmente en las nuevas estructuras de hospedaje y alimentación, minimizando los impactos negativos sobre el medio ambiente. Las urbanas y rurales, inteligentemente han potencializado sus recursos, que son revertidos en mejoras de carreteras, infraestructura y por supuesto en mejorar la atención de los servicios de calidad, marco programado para evitar la incertidumbre en la proyección de la actividad turística, considera la uniformidad con que se quiere manejar cierta tipología de turismo sin un previo diagnóstico geográfico, económico, medioambiental y psicosocial.

El Ecuador cuenta con indicadores de privilegio en relación a esta planeación turística que regenera la actividad del viajero en múltiples destinos nacionales dentro de sus cuatro regiones, privilegia los conocimientos ancestrales y las fuerzas de la naturaleza, que deben y tienen que ser gobernadas por líneas estratégicas de planificación, se mantienen los planes, programas y proyectos articulados entre sí, se busca la ejecución y operacionalización de las actividades previamente organizadas, vinculando en puntos claves a las instituciones que tienen que ver con la planificación del turismo en el país.

La inversión de las empresas privadas, nacionales y extranjeras, regenera y oxigena la innovación de nuevos servicios en comodidad y calidad armonizados con el entorno y encumbrando a una permanencia en el mercado a largo plazo. Las instituciones gubernamentales se estructuran en múltiples apoyos de colaboración y desarrollo logístico, interactuando entre ellas por un bien común de proyección turística nacional e internacional. 
Las Instituciones de Educación Superior (IES) vigilantes de los nuevas tendencias y mega tendencias turísticas globalmente hablando, planifican sus currículos motivados por el despliegue intelectual de los futuros profesionales, en particular en el desarrollo cognoscitivo y metacognitivo, en el rol de la cientificidad turística y el comportamiento profesional dentro de la sociedad sin caer en mediocridades endebles a la corrupción, etiqueta de crecimiento en su libertad de pensamiento, su ideal crítico y su compromiso en la aplicación de los principios éticos y morales. Los escenarios sociales a veces no son muy alentadores por la desesperación poblacional de crecer económicamente, mientras que el turismo espera y construye con paciencia esos emprendedores programáticos de nuevos servicios sin descuidar la calidad en la atención al viajero, esas necesidades son conjugadas con actividades complementarias para atraer a los potenciales clientes fijos en esta integral planificación.

La transformación, el diseño, producción y distribución de bienes, servicios, productos y procesos, para satisfacer necesidades sociales, requieren de un cónclave de operaciones estratégicamente imbricadas y realizadas con exactitud, permite la representación lógica de resultados óptimos con estimaciones ventajosas para un fin en común, representadas en la recopilación, tabulación, análisis, síntesis, interpretación y comparación de información diagnóstica sobre el elemento investigado (Rivera, Oviedo \& Galarza , 2016).

La planificación es el proceso de establecer metas y elegir medios para alcanzar dichas metas (Ortiz, M. 2018). Es el paso que se sigue para determinar en forma exacta lo que la organización hará para alcanzar sus objetivos. Es el asunto de evaluar toda la información relevante y los desarrollos futuros probables, da como resultado un curso de acción recomendado: un plan (González, Y. 2015).

Es el trascurso de establecer objetivos y escoger el medio más apropiado para el logro de los mismos antes de emprender la acción (Herrera, M. 2014). Según la definición propuesta por Jiménez, A. (2016), señala: "La planificación es un proceso de toma de decisiones para alcanzar un futuro deseado, se tiene en cuenta la situación actual y los factores internos y externos que pueda influir en el logro de los objetivos"

La planificación turística es un proceso que escucha las necesidades vulnerables de la naturaleza y a todos sus elementos, prioriza soluciones, enraíza compromisos, valora aportes y enaltece la participación de la especie superior que garantiza la contemplación de los recursos por mucho tiempo (Rivera, Oviedo \& Galarza, 2016).

Estos autores consideran a la planificación como un proceso que busca relacionar acciones y operaciones interinstitucionales, para alcanzar metas y objetivos, que organicen y otorguen racionalidad en el uso de los medios de producción y los patrones de consumo sobre los recursos que algunas veces son escasos y otros son limitados.

\section{Pasos para la Planificación}

Según Sánchez (2007), generalmente se definen varios aspectos relacionados con los pasos para lograr una buena planificación, entre estos podemos señalar los siguientes:

- Detección de una oportunidad 
- Establecimiento de objetivos

- Consideración de las premisas de la planificación

- Identificación de las alternativas

- Comparación de alternativas de acuerdo a los objetivos y las metas

- Elección de una alternativa

- Elaboración de los planes de apoyo

- Elaboración del presupuesto

La planificación a priori forma parte ineludible del diario hacer particularmente de las masas populares que vinculan su accionar a una jerga de comportamientos eyaculatorios de facilismo y mínimo esfuerzo, se forjan únicamente problemas y compromisos, sociales, económicos y ambientales sino se trabaja de manera integral, identificando el problema y su planteamiento siendo un primer paso funcional para su posterior solución, mediante objetivos factibles que priorizan las necesidades identificando alternativas en correspondencia a los objetivos planteados con anterioridad vinculando las metas accesibles a las alternativas configuradas en planes de apoyo y enrutadas en recursos financieros, humanos, económicos, tecnológicos entre otros, sería difícil alcanzar el éxito en un nuevo negocio turístico.

Organizar, con anterioridad las actividades que se realizaran, es decir ampliar las posibilidades de éxito en los diferentes ámbitos sociales, buscar con anterioridad las posibles soluciones a problemas identificados y priorizados con antelación. Buscar y utilizar los recursos eficaz y eficientemente para la solución de problemas, es otro limitante de la planificación social que desvirtúa sus capacidades innatas, por carecer de pensamiento periférico que proporciona el método de observación.

La evolución de la planificación turística. La relatividad del tiempo es visible en todos los aspectos naturales en que el hombre tiene injerencia. Los cambios ideológicos han generado teorías y sistemas de desarrollo económico y turístico que no son infalibles por los entornos socio-culturales propios de la cosmovisión local, pero son alcanzables de acuerdo a los compromisos humanos. Los múltiples acontecimientos mundiales que son reflejados en las financieras teorías políticas y económicas en las cuales se enfrascan los países desarrollados, de economías emergentes, en vías de desarrollo y subdesarrollados, hacen que el planeta reniegue de su equilibrio cíclico, puesto en manos del hombre que cambia a su antojo por más simple que parezca.

\section{Niveles de Planificación}

Según, Mora, Vera \& Melgarejo (2015), Establecen tres niveles de planificación:

A corto plazo.- Se refiere a adoptar medidas destinadas a mejorar la variación estacional de la demanda o la adaptación a los cambios coyunturales que puedan presentarse en el mercado o en la prestación de los servicios turísticos. 
A mediano plazo.- Es posible efectuar cambios en la infraestructura y el equipamiento de maquinaria, facilitando el cambio estructural del producto turístico.

A largo plazo.- Son decisiones que están relacionadas con la expansión de la actividad turística en su conjunto, es decir son las medidas y decisiones que harán sentir sus efectos en el futuro.

El turismo ha evolucionado en diferentes áreas geográficas, con las múltiples características que esta actividad produce, los posibles proyectos, la inversión que se requiere para su buen funcionamiento y por supuesto mejorar íntegramente la relación hombre - naturaleza. Como fuente de riqueza el turismo ha ampliado sus productos, procesos, bienes y servicios en beneficio del viajero y de los prestadores de actividades de recreación y distracción, evidenciando su evolución en nuevos destinos turísticos a gran nivel y sin escatimar lujos y comodidades extravagantes, lograr nuevas ideas de servicios no se podría realizar sin un entramado de ingenierías doctas a cambios y exigencias humanas mediante nuevos procedimientos y procesos que, se consolidan por la importancia de alcanzar objetivos y metas a corto, mediano y largo plazo, conformando una tendencia lógica de éxito por la persistencia y proyección de acciones sujetas a cambios.

\section{Componentes de la Planificación.}

Para, Hernández, N., González, S y Muñoz, P. (2014). La planificación turística pretende integrar a todos los elementos de la planificación turística de una región anclados en el financiamiento de las empresas privadas en movilidad de capitales nacionales y extranjeros, la motivación a las inversiones en prevención a cambios estructurales económicos, sociales y ambientales formulando planes de contingencia que asuren el desenvolvimiento racional de los viajeros en plena respuesta a la estructura sistemática de los componentes de la planificación: gestión, previsión, plan, organización, integrando corresponsabilidades de seguridad, complacencia, compromiso y solidaridad en función de cambios valorativos poblacionales como: puntualidad en las operaciones turísticas, amabilidad en la asistencia a los viajeros, respeto y responsabilidad comunicacional y generosidad en el apego a los pagos preestablecidos por servicios recibidos en los diferentes niveles de organización instrumental.

La aportación de Santana,(2012), está en los niveles de una organización donde se puede considerar:

- Estratégica. - Proceso realizado a largo plazo, plantea objetivos de carácter específicos se parte de la premisa de orden interno y externo

- Táctica. - Conjunto de acciones continuas y permanentes, que aclara la toma de decisiones y posteriormente son medibles y evaluables.

- Operativa. - Es el nivel donde participan los empleados, esta es la parte operativa de la empresa turística.

- Normativa. - Es el proceso que considera las políticas, normas y reglas para 
el buen funcionamiento de la institución se insiste en alcanzar los más altos niveles de estándares, en sus métodos y en la misma metodología.

\section{Estilos de Planeación}

Russell y Ackoff, (2002) identifican en la planeación cuatro actitudes generales, que dependen del tipo de enfoque que se le otorgue. Estas actitudes pueden encontrarse mezcladas en diversas proporciones dentro de una organización, y pueden variar según la situación, entre estos podemos señalar:

- Inactiva. - es restringida en la planeación debido a que considera infructuoso cambiar la situación actual, debido a que la empresa se encuentra en equilibrio, estabilidad y supervivencia

- Reactiva. - es la visión de túnel que tradicionalmente se preocupa de inoperar nuevas acciones se mantiene en la manera arcaica de planificar sin aperturarse a las nuevas tecnologías.

- Proactiva. - son proyecciones futuristas que permiten optimizar las oportunidades de mercado, se anticipan los posibles proyectos de mejora para la empresa turística.

- Interactiva. - es la proyección que se realiza desde las acciones y decisiones tomadas en el presente, se previene holgadamente las futuras crisis.

Los niveles de planificación turística responden a problemáticas que obstaculizan el desarrollo de iniciativas de recreación y entretenimiento para el viajero o visitante en una región, se contempla desde luego esos múltiples intereses nacionales e internacionales como: costos, seguridad, alimentación, hospedaje, diversión, transporte, oferta, demanda, entre otros. Esto implica determinar la oferta que el país tiene para las diferentes actividades turísticas que se pueden realizar en el país, se direcciona contemplativamente las nuevas tendencias de los grupos de viajeros y sus demandas, que son organizadas y articuladas potencialmente para el disfrute de los involucrados. La actividad turística tiene características relacionadas con las economías especialmente atractivas: es una industria exportadora de primer orden con una gran capacidad de generar divisas; es un sector intensivo en empleo; la inversión y capacitación productiva necesarias para el desarrollo de un destino, resultan menos gravosas que las necesarias para otras actividades, pero, ¿es el turismo sólo un sector económico?, ¿es posible circunscribir todo lo que conlleva el fenómeno turístico a los impactos económicos que genera, por muy importantes que estos sean? Se reconoce que la dimensión económica es clave para entender las dinámicas que genera, el turismo es más que un sector de la economía (Velasco, 2013)

El desarrollo económico de una nación se coordina con el turismo desde la autoridad, que multiplica acciones en los destinos turísticos para fortalecer las propuestas públicas y privadas de actores que sostienen la misma planificación territorial traducida en sostenibilidad donde el geocentrismo de la naturaleza son la temperatura, el clima, las especies, la lluvia, el agua, el viento, la reproducción, el nacimiento, la transformación, la metamorfosis, la contemplación entre otros. Todo esto inspira la elaboración de herramientas 
e instrumentos operativos y reguladores de esta actividad para su mejor desenvolvimiento de servicios, bienes y procesos turísticos. Este proceso se tiene que alcanzar por etapas óptimas de planificación con orden, compromiso y seguridad, identifica responsabilidades de cumplimiento en lo político, social, económico, cultural, ecológico y espiritual, entiéndase espiritual desde los valores éticos poblacionales y gubernamentales, proporciona el equilibrio necesario en la mejora de la calidad de vida de la población y el disfrute racional de nuestros recursos naturales y culturales. Esta planificación estaría incompleta si olvidamos los recursos financieros, tecnológicos, humanos, la evaluación y su seguimiento, plasmados en programas, presupuesto e innovación en los objetivos delimitados.

\section{Importancia de la Planificación Turística}

La planificación turística es importante por la organización y operación de actividades y acciones, según sus diferentes factores descritos en el proceso, que mentaliza diferentes áreas de desarrollo, según, Cordero, J. (2009):

En el área económica, es lógico buscar el desarrollo del destino, impulsando la oferta, demanda en beneficio del mercado meta, implementando nuevos pymes en apoyo a la innovación y mejoramiento de los bienes y servicios, que fortalece la inversión extranjera y facilita el incremento de visitas, esta área económica está al tanto de los gatos y presupuestos que solidifican las alternativas de desarrollo por encima de la satisfacción de las necesidades del viajero.

En el área social se minimizan los rasgos psicológicos controlando la incertidumbre del mercado con las proyecciones futuristas que encadena el aprovechamiento de oportunidades en la atención con eficiencia en menor tiempo y esfuerzo que reduce las acciones hostiles en el mercado y el producto.

En el área política fijarse niveles tope en las garantías de contingencia racionalizando la toma de decisiones en fiel cumplimiento de las normativas estatales y evaluando a posteriori el avance de las alternativas.

En el área cultural estimular la preparación académica, el rescate de costumbres y tradiciones, enriquecidas con el intercambio de experiencias populares.

En el área ambiental se debe condicionar la estructura para actividad turística, en fiel cumplimiento de la normativa para minimizar los impactos ambientales sobre el medio natural en auspiciante uso racional de los recursos, adaptando las actividades al compromiso comunicacional con el macro y micro cosmos.

En el área ética es necesario se practique la gobernanza y la gobernabilidad, incrementar el valor agregado en los recursos culturales, particularmente en los saberes ancestrales.

La importancia de la planificación en el turismo se reduce a la transversalidad, sine qua non, del mejoramiento sustancial y significativo de la calidad de vida poblacional se estiman sus necesidades en logros grupales. Intereses particulares, que agotan y agobian estos talentos, 
no encuentran satisfacción en el trabajo, que maximiza potencialmente emprendimientos turísticos de gran calado nacional e internacional.

Abordar el tema de la importancia de la planificación turística es una acción orientada a analizar los fundamentos conceptuales y categoriales de la planificación turística a efecto de generar una visión lógica y holística del proceso. Parte de este análisis nos ayudará a sintetizar el proceso básico de planificación turística aplicable en todos los niveles de planificación. Tiene que ver con la relevancia multidimensional que la actividad planificadora posee en turismo (gestiondedestinos.wordpress.com; 2011)

\section{Ventajas de la Planificación Turística}

El autor Gunn, C. (1994), Interioriza las ventajas de la planificación turística con variables de integración compartamental como: Integra los multiniveles de planificación de manera geométrica, ayuda a la adaptabilidad con el medio ambiente buscando la sustentabilidad a largo plazo, permite operar y ejecutar las actividades, facilita la navegación turística de manera global, articulación de bienes, servicios y procesos turísticos, mejora y amplía la coordinación del equipo multidisciplinar basando experiencias en objetivos comunes, minimiza conflictos y mejora la visión del emprendimiento turístico a mediano plazo.

La ventaja que realza este tipo de planificación es la integración de multiniveles operativos nacionales y seccionales con sus respectivas competencias. La integración multidisciplinar, es decir configurar los aportes de profesionales que aúnan esfuerzos en un objetivo común de desarrollo turístico poblacional, es la ventaja más relevante desde la organización de proyectos turísticos, con esquemas futuristas de varias promociones como "ECUADOR AMA LA VIDA", "ALL YOU NEED IS ECUADOR", "HAPPY IN ECUADOR", que sistematiza la oferta de un país con múltiples alternativas de disfrute y distracción.

La evidencia empírica recomienda no plantear la planificación territorial del Turismo desde una perspectiva puramente sectorial o meramente territorial, es decir sin tener en cuenta a otros sectores económicos (agropecuario, agroindustrial, comercio, servicios, construcción, infraestructuras o transportes) las comunidades locales, los agentes políticos e institucionales, o los agentes socioeconómicos territoriales, en definitiva la planificación no puede omitir a la realidad territorial. Tampoco puede eludir a las propias empresas turísticas que son el agente básico de desarrollo turístico. Si las condiciones previas no son atendidas, podría significar la irrealidad de la planificación y su consiguiente inaplicación, cosa por desgracia bastante frecuente y que explica, aunque no justifica, ese cierto descrédito de los planes de desarrollo.

Los autores Mill y Morrison, (2012) manifiestan que existen cinco objetivos básicos buscados por la planificación turística, estos son:

- Identificar las alternativas de desarrollo y organización de la empresa y sus actividades

- Adaptarse a los cambios del macro y micro entorno.

- Mantener o buscar la diferencia en recursos naturales, culturales, arquitectónicos, otros 
- Crear alta rentabilidad e imagen positiva.

- Evitar situaciones desagradables, como la destrucción y la alteración del medio ambiente, polución, actitudes hostiles por parte de los residentes ante la llegada de turistas.

\section{Servicios Turísticos}

Los servicios turísticos responden siempre a la satisfacción de necesidades básicas de los turistas: hospedaje, alimentación, transporte e información turística clave. Los servicios son un conjunto de bienes, productos y procesos, que integrados entre si forman parte de la oferta turística de una zona geográfica local, nacional, regional e internacional. Lo más importante en las necesidades del turista es ser atendido con mucha hospitalidad, esto produce en los interlocutores una empatía que interrelaciona oferta y demanda en sus niveles más incluyentes de los seres humanos.

El cumplimiento de estándares de calidad en los servicios se puede medir con diferentes indicadores como: procedencia del turista, número de visitantes al año o en fechas tradicionales, las divisas utilizadas en sus transacciones, los lugares más visitados, entre otros, indicadores que permiten mejorar según las exigencias de la nueva ola de turistas nacionales y extranjeros.

Desde una óptima muy personal los servicios turísticos parten al unísono de la atención que se brinda al contingente de visitantes, la primera impresión en territorio es la hospitalidad, en Ecuador esto se reduce estrechamente al entramado de procesos en la atención vinculado con la amabilidad inequívoca muestra de los ofertantes. En la mayoría de establecimientos que brindan servicios se muestra la cultura del "vuelva pronto por favor, los esperamos", pero en otros se conserva la exagerada jovialidad en los tratos. Sin incluir los precios exorbitantes y que no cumplen una relación de producto y servicio, enmudeciendo el turismo justo, turismo equitativo y turismo responsable, turismo solidario (Palomo, 2006).

\section{Metodología}

Este artículo estudia la integración de los elementos de la planificación turística desde el cumplimiento de la funcionalidad de sus procesos organizativos, ensambladas en la organización a priori de los objetivos, metas, objetos, principios y funciones pre establecidas en un conjunto de actividades a desarrollar, entre esas actividades están los servicios turísticos ofertas y su fiel cumplimiento en tiempos y productos. Para el estudio se aplicaron encuestas para relucir las necesidades insatisfechas del consumidor y la calidad de cada uno de los procesos planificados incumplidos.

\section{Resultados}

El año 2018 arrancó con fuerza en lo que se refiere al turismo internacional. Las llegadas de turistas internacionales aumentaron un 5\% entre enero y abril de 2018 según el último Barómetro de la Organización Mundial del Turismo (OMT, 2016). Los resultados fueron 
claramente buenos en casi todas las subregiones y muchos destinos registraron tasas de crecimiento de dos dígitos. Las perspectivas para mayo-agosto son también positivas, previéndose que en este periodo vacacional que representa la temporada alta para el hemisferio Norte, alrededor de 500 millones de turistas viajarán al extranjero.

Los destinos de todo el mundo recibieron 348 millones de turistas internacionales (visitantes que pernoctan) entre enero y abril de 2016, es decir, unos 18 millones más que en el mismo periodo del pasado año (5,3\%). Este crecimiento se suma a un aumento del 4,6\% en 2015, y podría hacer de 2016 el séptimo año consecutivo de crecimiento superior a la media, dándose así la circunstancia de que desde 2009, el año de la crisis, las llegadas de turistas internacionales han aumentado un $4 \%$ o más cada año.

«Los resultados muestran un firme deseo de viajar y ese deseo sigue impulsando el crecimiento del turismo. La demanda en todas las regiones del mundo sigue siendo sólida, a pesar de los desafíos presentes, lo que demuestra que el turismo es un sector económico dinámico y resistente», afirmó el Secretario General de la OMT.

«No obstante, y a pesar de estos buenos resultados, los trágicos sucesos de los últimos meses nos recuerdan que la seguridad siendo un reto mayúsculo para todos. Debemos seguir colaborando estrechamente para enfrentarnos a esta amenaza global y garantizar que el turismo forme parte integral de todo plan de emergencia o respuesta a escala mundial, regional y nacional».

Por regiones, Asia y el Pacífico (+9\%) fue la región con un mayor incremento de las llegadas de turistas internacionales, habiéndose registrado en todas las subregiones asiáticas una tasa de crecimiento del 7\% o superior. Por subregiones, el África Subsahariana (+13\%) se situó a la cabeza del crecimiento, recuperándose con fuerza de los modestos resultados de los años anteriores.

La OMT (2016) prevé que las llegadas de turistas internacionales aumentarán entre un 3,5\% y un 4,5\% a lo largo del año 2016, confirmándose la previsión a largo plazo realizada por la OMT y que apuntaba a un crecimiento del 3,8\% anual entre 2010 y 2020.

\section{Discusión}

Materializar la visión empresarial turística constituye configurar algunos elementos complementarios que garantizan el desarrollo óptimo del destino, se parte de los objetivos planificados con antelación, y por supuesto el uso racional y equilibrado de los recursos. La competencia desorganizada de ofertas manipula el verdadero sentido de disfrute y descanso del viajero, implementar paquetes turísticos que manejen una relación de confraternidad natural y cultural, se beneficia y se establece un mercado base, de conservación y protección. Para ejecutar la planificación es crucial planificar para el desarrollo y cambio de comportamiento en la atención al cliente, se deben extender objetivos transversales en la misma planificación turística nacional. Estas transformaciones del hombre sobre el entorno conllevan el abuso de más recursos y la presión sobre los elementos naturales, aunque tratemos de minimizar sus impactos son sentidos de manera perceptible en todo el planeta: ¿se planifica para la destrucción?, esta es una pregunta que requiere respuestas inmediatas. 


\section{Conclusiones}

Antes de emprender cualquier proyecto de desarrollo turístico debemos proceder a su evaluación se tiene en cuenta los resultados económicos, los culturales, sociales y capacidad de carga.

Es fundamental la planificación para que, avance el futuro y gestione los impactos de la actividad turística en el territorio, es un desenlace que se juzgara a posteriori con resultados incluidos en el macro contexto de desarrollo y proyección turística. La planificación tiene que partir de la sostenibilidad como principio informador en la implantación y desarrollo de la actividad turística.

Existen zonas que cuentan con potencialidades turísticas: agroturismo, ecoturismo, turismo cultural, turismo de negocios, turismo de salud, turismo gastronómico, turismo de aventura y turismo comunitario, estas tendencias abren la oportunidad de incursionar tentativamente en el nuevo mercado de actividades para viajeros que buscan el contacto directo con la naturaleza.

La debilidad de la planificación turística en algún sector geográfico de un país implica, la desintegración de actividades en los elementos de la planificación que sobrepasa puntos extremos de desinterés en las empresas públicas y privadas: y su posterior abandono.

Los servicios turísticos acompañados de la calidad en la atención a los viajeros es una línea vertical de compromiso y sostenibilidad para las actividades complementarias dentro de un área aritmética o geométrica de desarrollo, las tendencias y mega tendencias responden a este fin como lo más importante en materia de satisfacción bidireccional de complacencia.

Es necesario realizar un estudio del impacto ambiental y social del proyecto turístico para identificar la fragilidad social, ecológica del territorio y las posibles acciones a tomar.

\section{REFERENCIAS BIBLIOGRÁFICAS}

Cordero, J. (2009). Comunidad receptora: Elemento esencial en la gestión turística. Gestión turística, (11), 101-111.

Gunn, C. (1994) Tourism Planning (3a edición). Nueva York, Taylor and Francis

Russell J., Ackoff, R. (2002). El paradigma de Ackoff, una administración sistémica. México. Editorial Limusa. González, Y. P. (2015). La planificación empresarial: un acercamiento conceptual. Recuperado el.

Hernández, N., González, M., \& Muñoz, P. (2014). La planificación del aprendizaje colaborativo en entornos virtuales. Comunicar, 21(42), 25-33.

Herrera, I. (2014). La planificación en el contexto administrativo. Venezuela.

Mill, R., \& Morrison, A. (2012). The tourism system (Séptima ed.). Electronic Delivery EBOOK.

Mora Riapira, E. H., Vera Colina, M. A., \& Melgarejo Molina, Z. A. (2015). Planificación estratégica y niveles de competitividad de las Mipymes del sector comercio en Bogotá. Estudios gerenciales, 31(134), 79-87. 
Organización Mundial del Turismo, (OMT, 2016). El turismo internacional continúa creciendo por encima de la media en los cuatro primeros meses de 2016. Barcelona - España: Organización Mundial de Turismo.

Palomo, S. (2006). El turismo justo y la creación de sistemas de producción de servicios turísticos responsables. Estudios Turísticos No. 168, pp. 7-46

Sánchez, N. (2007). El marco lógico. Metodología para la planificación, seguimiento y evaluación de proyectos. Visión gerencial, (2), 328-343.

Rivera García, C., Oviedo Rodríguez, M., \& Galarza Bravo, F. (2016). Visión y Planeación turística; Responsabilidad 100\%Humana. Babahoyo: Los autores - Imprenta Don Gonzalo.

Santana, C. (2012). Los niveles de gestión en una organización. Bogotá - Colombia: Universidad de los Andes.

Velasco González, M. (2013). Gestión pública del turismo. La gobernanza. Madrid - España: Universidad Complutense de Madrid. 


\section{PARA CITAR EL ARTÍCULO INDEXADO}

Rivera García, C., \& Cachipuendo Castillo, J. (2019). La Planificación turística, enfoque de la calidad en los servicios. Visionario Digital, 3(4), 67-80.

https://doi.org/10.33262/visionariodigital.v3i4.970

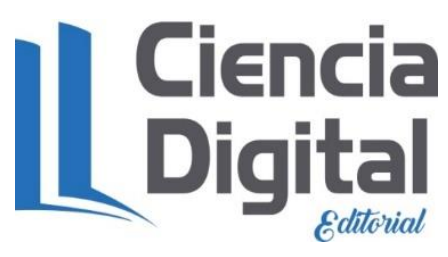

El artículo que se publica es de exclusiva responsabilidad de los autores y no necesariamente reflejan el pensamiento de la Revista Visionario Digital.

El artículo queda en propiedad de la revista y, por tanto, su publicación parcial y/o total en otro medio tiene que ser autorizado por el director de la Revista Visionario Digital.

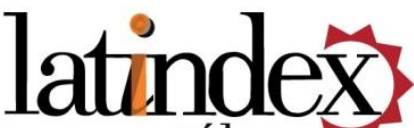

catálogo

0

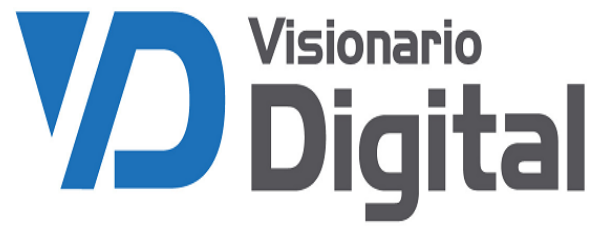

